\title{
Availability modeling approach for future circular colliders based on the LHC operation experience
}

\author{
Arto Niemi, Andrea Apollonio, Johannes Gutleber, and Peter Sollander \\ CERN - The European Organization for Nuclear Research, CH-1211 Geneva, Switzerland \\ Jussi-Pekka Penttinen \\ Ramentor Oy, Hermiankatu 8 D FI-33720 Tampere, Finland \\ Seppo Virtanen \\ Tampere University of Technology, PO Box 527, FI-33101 Tampere, Finland
}

(Received 19 July 2016; published 27 December 2016)

\begin{abstract}
Reaching the challenging integrated luminosity production goals of a future circular hadron collider (FCC-hh) and high luminosity LHC (HL-LHC) requires a thorough understanding of today's most powerful high energy physics research infrastructure, the LHC accelerator complex at CERN. FCC-hh, a 4 times larger collider ring aims at delivering 10-20 ab $\mathrm{ab}^{-1}$ of integrated luminosity at 7 times higher collision energy. Since the identification of the key factors that impact availability and cost is far from obvious, a dedicated activity has been launched in the frame of the future circular collider study to develop models to study possible ways to optimize accelerator availability. This paper introduces the FCC reliability and availability study, which takes a fresh new look at assessing and modeling reliability and availability of particle accelerator infrastructures. The paper presents a probabilistic approach for Monte Carlo simulation of the machine operational cycle, schedule and availability for physics. The approach is based on bestpractice, industrially applied reliability analysis methods. It relies on failure rate and repair time distributions to calculate impacts on availability. The main source of information for the study is coming from CERN accelerator operation and maintenance data. Recent improvements in LHC failure tracking help improving the accuracy of modeling of LHC performance. The model accuracy and prediction capabilities are discussed by comparing obtained results with past LHC operational data.
\end{abstract}

DOI: 10.1103/PhysRevAccelBeams.19.121003

\section{INTRODUCTION}

The potential for new discoveries in high energy physics relies on production of a sufficient number of particle collisions over an extended period of time. This requires high availability of the particle collider complex as it needs to produce collisions under well-known conditions for multiple operational years. With a growing dominance of operation costs for large-scale research infrastructures, improving overall availability has moved into the spotlight of attention.

It is mandatory to achieve a thorough understanding of an operating accelerator complex, to develop a model that can reproduce past and present performance with high accuracy. Only such a model can permit simulating the performance of a future energy frontier collider.

The international future circular collider collaboration studies conceptual designs for proton-proton and

\footnotetext{
* Corresponding author. arto.niemi@cern.ch

Published by the American Physical Society under the terms of the Creative Commons Attribution 3.0 License. Further distribution of this work must maintain attribution to the author $(s)$ and the published article's title, journal citation, and DOI.
}

electron-positron high-energy frontier machines in a global context. Already at the kick-off meeting of the study [1], it became clear that meaningful and credible reliability and availability goals need to be set for such a largescale particle collider, to ensure feasible operations [2]. Successes in LHC and HL-LHC availability studies [3,4] encouraged our model based approach for defining these goals. However, this approach will rely on operational data collection over multiple years to have credible results. For the LHC availability of this type of data is currently limited, but efforts to improve accelerator fault tracking are ongoing at CERN [5]. Thus, this paper presents only the concept of the approach and validating results with LHC operational data.

Our availability study establishes and continuously refines a model of CERN's LHC complex (see Fig. 1), applying established methods and tools, which have turned out to be effective for large-scale industries [6-9]. This novel approach combines industry best-practices with tracking of an evolving machine operation, taking into account beam-related parameters and the relation to overall availability for physics data taking. The basis for the analysis are monitoring and logging data from LHC run 1 (2010-2012), current and future operation. The 


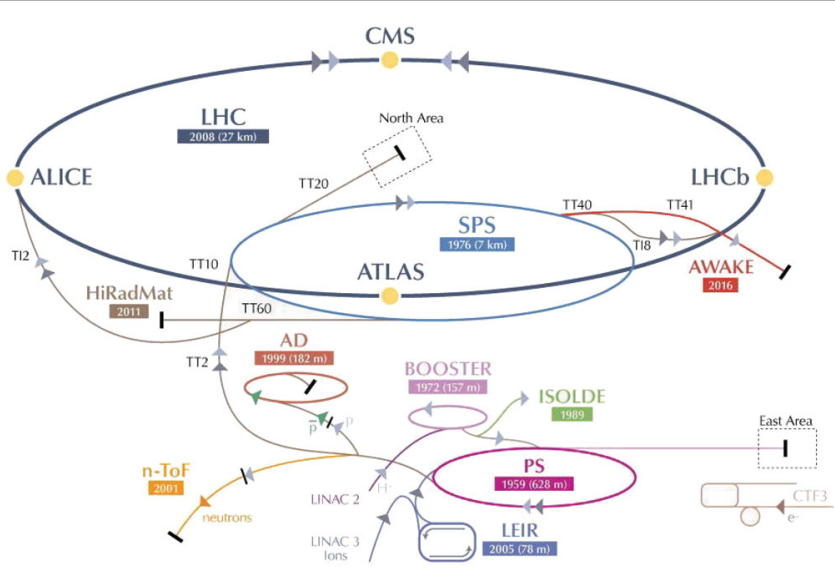

FIG. 1. CERN's accelerator complex [11].

approach has potential applications to other particle accelerator infrastructures. Light sources, ion-therapy facilities, accelerator-driven nuclear energy systems, neutron sources and other ion-beam based research accelerators have an ever growing need to improve their value to cost ratio. As a matter of fact, the research and development around reliability and availability in the scope of the FCC study has found its way into the ARIES H2020 project (Accelerator Research and Innovation for European Science and Society) [10]. In the scope of this project also the synchrotron-based Heidelberg Ion Therapy (HIT) facility, now operating also the Marburg treatment facility, cooperates with CERN on establishing a common database of subsystem and system failure distributions, reliability and availability modeling, and simulation-based sensitivity analysis.

For particle colliders aiming at fundamental physics research, the production of integrated luminosity is the key performance indicator (KPI), being a measure for the number of events potentially observed by the particle detectors.

$$
L_{\text {int }}=\int_{0}^{t} L(t) d t
$$

where $L(t)$ is instantaneous luminosity, which gives the rate of events for a given time $t$ for interactions with unit cross section [12].

Maximizing the integrated luminosity production depends on the time with colliding beams of adequate quality, which in turn depends on the availability of the entire accelerator infrastructure [3]. All accelerators and their operation cycles along the chain need to be included in the model, since the operational efficiency of each machine in the injector complex affects the luminosity production of the collider.

Here, we introduce a viable method to model cyclic beam production processes, generalized to all types of particle accelerators. We simulate the operation of the accelerator complex using a generic stochastic model that combines semi-Markov Chains [13] with dynamic fault trees [14]. Based on our survey (Sec. II C) this approach is novel in accelerator availability modeling. The model and Monte Carlo simulation approach can be applied to arbitrary particle accelerators, independent of system size (number of subsystems and components), complication (number of different elements interacting), and complexity (degree of interrelation and emerging behaviour).

This paper describes the model and presents initial results from the validation of the concept using 2012 operation data from CERN's LHC complex. In addition, we demonstrate the model's prediction capabilities and we describe a sensitivity analysis example to show that value of the approach. The paper sheds light on the challenges to establish a scalable model for an entire accelerator complex, devising the reliability distributions for the elements in the individual fault trees and outlines how this successful approach will be applied to the HL-LHC design and feasibility assessment of a post-LHC energy-frontier hadron collider.

\section{SURVEY OF RELIABILITY MODELING METHODS}

\section{A. Definitions}

This section provides a short introduction and provides references to reliability modeling concepts and methods cited in this paper. Reliability is defined as the probability that a system performs as required for a given time interval $\left(t_{1}, t_{2}\right)$, under defined conditions [15]. Availability is defined as percentage of time when the system is able to be in a state to perform as required.

In this context we define availability as the percentage of time when the collider produces luminosity.

Availability for physics $=\frac{\text { Luminosity production time }}{\text { Operation time }}$.

Different availability criteria exist: for example, the operation time can refer to the entire collider life cycle spanning the time from commissioning to decommissioning or it may be limited to the time reserved for physics operation. Different interpretations of availability complicate comparisons of achieved performance between facilities and different systems.

Our work addresses this issue by introducing a system production function as opposed to a dimensionless availability indicator. For different accelerators in the complex, the production function looks different. In our case, the function of the particle collider refers to the production of integrated luminosity measured in inverse femtobarn. The production function can be used to form for other KPIs, such as cost per production unit or overall equipment 
efficiency (OEE), that compares achieved production to the theoretical maximum production [16]. Different colliders have different luminosity production functions. For LHC functions in [17] accurately describe the integrated luminosity production [3]. For FCC-hh [18] introduces the analytical expressions for integrated luminosity production. For injectors production function measures the number and quality of delivered bunches.

\section{B. Modeling approaches}

State-of-the-art reliability modeling methods distinguish qualitative and quantitative methods: while qualitative methods are applied to investigate the effects of faults and failures, quantitative methods permit forecasting reliability and availability. The failure modes and effects analysis (FMEA) [19] is a systematic, qualitative bottom-up approach to identify failure modes and their effects. The fault tree analyses (FTA) [20] and reliability block diagrams (RBD) [21] are deductive top down methods used to determine the causes for specific faults. These can be used both qualitatively and quantitatively. A fault tree analysis starts by determining a top event (undesired critical event). The tree is developed to identify various parallel and sequential combinations of faults that will result in the occurrence of the top event. RBDs are success oriented networks that model logical connections of components needed to fulfill the system function.

The quantitative methods depend on operation data and expert estimates on failure rates and repair times. In case analysis is based on operational data, first, the data needs to be collected and assigned to the relevant subsystems. Next, the data needs to be mapped to the failure modes of the subsystems in that model. Then, statistical functions are used to describe the observed failure and recovery characteristics. The failure behavior of typical systems has been described in literature [22] and characterized by wellestablished distributions (e.g. exponential, log-normal, Weibull).

Markov chains can be used to model the transitions between system states (e.g. different operational phases and failures). If the transition probabilities are constant (i.e. they follow exponential distribution), states form a Markov process that has an analytical solution [13]. An extension of the Markov process with nonconstant transition rates is called the semi-Markov process (SMP). A solution for such a process can be found applying numerical methods or discrete event simulation (DES). The DES is a Monte Carlo simulation method [7] that simulates each state change in the system as an event.

Qualitative FTA or RBD can be extended with the failure distributions to come to a model of the plant operation to determine the achievable reliability of the system. Adding also to each node information about the assumed maintenance and repair times permits system availability analyses. Assigning cost tags to the downtimes and maintenance actions provides then the basis for a decision support model.

\section{Survey of accelerator availability studies}

Cost-effectiveness and availability of particle accelerators have become consistently more important with tightening user requirements and increasing complexity of infrastructure [23]. However, establishing a reliability and availability assessment process based on a proven method, supported with long-term monitoring data in a continuously evolving environment, is challenging. Traditionally, the target performance of particle colliders is specified in terms of very high-level goals (beam parameters [24]) or as hardware performance [25], without a structured process to devise and link physics requirements to machine and operation requirements. Due to the constraints imposed by technical systems (e.g., pile up in detectors, limited possibilities to implement fault-tolerant designs) and resources (e.g., limitation of personnel with required experience, operation budget), increasing availability has become a main concern for the operation of existing and future accelerators. Examples include SNS [26], ILC [27], Myrrha [28], CLIC [29], IFMIF [30], Linac4 [31], HL-LHC [32], CepC [33] and ESS [34]. User-oriented facilities [35], such as synchrotron light sources [36,37] and medical accelerators [38] are aware of the importance of availability, but improvements are mainly driven by the highest impact failures observed in operations, rather than a systematic modeling and simulation analysis.

The number of components and systems that make up a particle accelerator, the dynamic behavior of the machine resulting from the interplay of the beam-line elements, the beam and the ancillary systems, and the impact of different maintenance and repair policies, call for a software tool assisted approach. The tools potentially permit a direct quantification of the impacts of different operational scenarios, hardware modifications and maintenance actions.

Both, commercial and custom software tools have been used in the past to model the availability of particle accelerators [39]. At FNAL, a $\mathrm{C}++$ discrete event simulation model was developed for Tevatron [40,41]. The tool allowed modeling the luminosity production based on varying operational parameters. In particular, downtime for the various parts of the complex was included as well as beam-related parameters like transfer efficiencies, emittance growths and changes in the luminosity lifetime. This helped to determine and optimize operation strategies, and to forecast how improving reliability could improve the luminosity production.

For IFMIF, two different packages were evaluated $[42,43]$ before AvailSim was adopted $[30,44]$. This discrete event simulation programmed as a Matlab package was originally developed at SLAC in the framework of the ILC 
study [27,45]. In AvailSim [46] events represent failure modes of different components. Events affect functions that are used to represent degradation of the physical operational parameters, such as beam intensity or the system availability. Events can trigger the activation of further events. This permits modeling system dependencies and corrective maintenance actions after a failure. Additionally, the effects of the selected maintenance strategy and of the limited amount of repair personnel can be simulated. The tool is now evaluated in the frame of the ESS project. As the origins of AvailSim are for linear accelerator modeling, the concept of the production cycle (Sec. III A 3) has not been included. Our study shows that modeling this cycle is essential to model availability and luminosity production in circular colliders. Also the concept of operational schedules (Secs. III A 1 and III A 2) has not been included. This is an essential feature to optimize particle accelerator operation over multiyear periods.

In the preliminary design studies for the ADS concept PDS-XADS [47], reliability was studied using the RBD approach with the Relex software [48]. The software is currently known as PTC Windchill Quality Solutions. It allows fault tree analyses based on Monte Carlo simulations and specified failure mode and effect analysis and decision support tools that follow aviation, medical and car industry standards [49].

The Myrrha ADS study based a reliability forecast on fault-trees implemented in a commercial software called RiskSpectrum [50]. The model was parametrized using data from the operation of the SNS [51]. The software solves the minimum subset of components that can fail (minimal cut set [6]) and calculates analytically the probability for this set to fail.

For Linac4 [31] and a planned PS Booster upgrade [52] at CERN, Isograph [53], a commercial tool implementing Reliability Block Diagrams (RBD), was used to forecast the expected availability. With this tool, the user defines failure rate and repair time distributions and associate them to nodes in a fault tree or in a RBD. A Monte Carlo simulation engine activates the nodes according to the distributions. The tool also permits quantifying the impact of available resources (personnel and spare part stock) on the achieved availability and operation costs. The tool retrieves reliability figures for off-the-shelf electronic and mechanic components from a rich selection of standard handbooks, such as MIL-HDBK-217F [54].

A recurring obstacle to model and simulate accelerator availability is the lack of suitable subsystem reliability information derived from continuous operation data. The reason is two-fold: first, the number of particle accelerators with comparable operation conditions is small, and second, an integrated operation monitoring database considering operation phases, modes, and states remains to be established. An effort was launched during the 1990s to combine data from different machines $[43,55,56]$. However, the significant differences in machine hardware and operation limits the value of this approach [48,55]. Even similar accelerators in the world are operated differently. In addition, operation of a given accelerator might not be reproducible, due to continuous tuning of operating parameters

Our surveys revealed another substantial obstacle toward a unified approach to model, simulate, and assess availability of particle accelerators: the figures produced by different tools and working groups refer to different definitions of availability. In the case of SNS, the availability goal of $90 \%$ [26] refers to the beam generation, excluding the target operation performance. Consequently, the actual availability for beam production is different, although the accelerator indeed achieves over 90\% availability [57]. In recent development there is an attempt to standardize storage ring light source operation metrics [58].

\section{Practices established in industry}

Optimizing the cost-effectiveness of assets along their life cycle is a key requirement for industrial facilities. Methods have been developed a significant time ago to address reliability and availability along all phases of the life cycle of a system (see Fig. 2). Reliability centered maintenance (RCM) [59] is the established approach on which systematic methods and tools for reliability engineering and availability management have been developed: the governing principle is based on three elements: (1) establish component failure modes of a system; (2) determine the failure probabilities occurrence and consequences under different operation conditions; (3) assess the benefits and costs of component modifications, which result in an updated failure model.

RCM emerged from the aerospace industry in the late 1960s, revealing significant limitations in commercial aircraft availability due to reliability limitations and unnecessary planned maintenance. Consequently, the approach spilled over to the nuclear and manufacturing industries. The approach suits well in facilities that represent production processes consisting of several interlinked technical systems.

Modeling the reliability of large scale plants is today a common practice in industry. A representative example is the study of a chemical fertilizer plant operating with about 3000 employees, producing in 24/7 mode about $1500 \mathrm{t}$ of fertilizer per day [60]. This specific study has shown that a decision support model is essential to leverage the findings of the analysis and to be able to predict the benefits of the suggested improvements. Only continuous plant monitoring

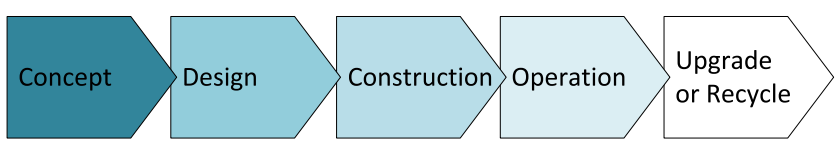

FIG. 2. System life cycle phases. 
can permit validating the effectiveness of the decision at a later stage.

We build on these findings: a particle accelerator complex can be seen as a beam production plant consisting of several interlinked systems (injectors, transfer lines, collider) that relies on numerous infrastructure systems, skilled operation personnel, and an effective maintenance concept. The following Sec. III sheds light on how the accelerator complex at CERN is operated and thus prepares the ground to explain our approach.

\section{COLLIDER OPERATIONS BASED ON LHC EXPERIENCE}

\section{A. Operation schedules}

\section{Multi-year operations}

Accelerators require periodic maintenance and consolidation actions to operate reliably. Over the life cycle of a particle accelerator complex, periods of luminosity production runs and maintenance shutdowns alternate. For example, the first run of the LHC lasted from 2010 to 2012 and the second is scheduled to last from 2015 to 2018 [61].

Within runs, the performance increases stepwise and approaches the original design parameters. To reproduce realistic operations this stepwise performance increase has to be modeled. The first year of LHC operation (2010) focused on machine commissioning. $45 \mathrm{pb}^{-1}$ of integrated luminosity were produced. In 2011 the commissioning was still ongoing and the production was $5.5 \mathrm{fb}^{-1}$. In 2012, the production was $23.27 \mathrm{fb}^{-1}$.

For LHC run 2 the bunch spacing was decreased from $50 \mathrm{~ns}$ to $25 \mathrm{~ns}$ and the collision energy was increased from $4 \mathrm{TeV}$ to $6.5 \mathrm{TeV}$. These changes, together with an improved availability, allowed increasing the luminosity production. In 2016, the LHC reached already $25 \mathrm{fb}^{-1}$ in August, surpassing the production estimates [62]. However, in 2015 the first year of run 2, the production was relatively low (about $4 \mathrm{fb}^{-1}$ ), due to machine recommissioning [63].

\section{Yearly operation modes}

In the current LHC operational strategy, planned maintenance is carried out at the end of each year extending to the beginning of the next, during the so-called year end technical stop (YETS). Three intermediate technical stops are also scheduled. The YETS is followed by machine commissioning periods to verify readiness for operation, separated into, (1) Hardware commissioning, to prepare technical systems for safe beam operations; (2) Beam commissioning with low intensity beams, with low damage potential. Then, luminosity production starts and the number of the bunches and the bunch intensity is progressively increased to reach the nominal parameters [64]. Figure 3 shows the intensity ramp-up in 2012 with the

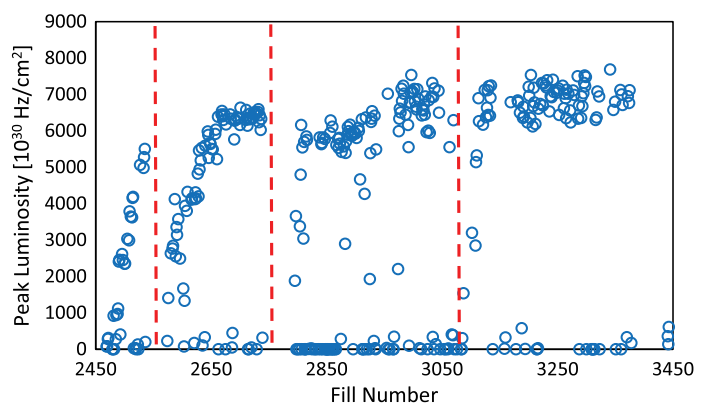

FIG. 3. Peak luminosities of fills during 2012. The lines indicate the technical stops [3].

evolving peak luminosity. A short intensity ramp-up is also carried out after each technical stop. Proton physics production is also interrupted for machine studies and other physics production modes. Figure 4 shows an example of the LHC schedule.

\section{Production cycle}

Figure 5 shows a typical operation cycle of a collider which is divided into phases: (1) Prepare injection: magnets are ramped up to injection field. (2) Injection: the rings are filled with beams injected from the injector chain. During this phase, the collider depends on the injector performance. (3) Ramp-up: the beams are accelerated to collision
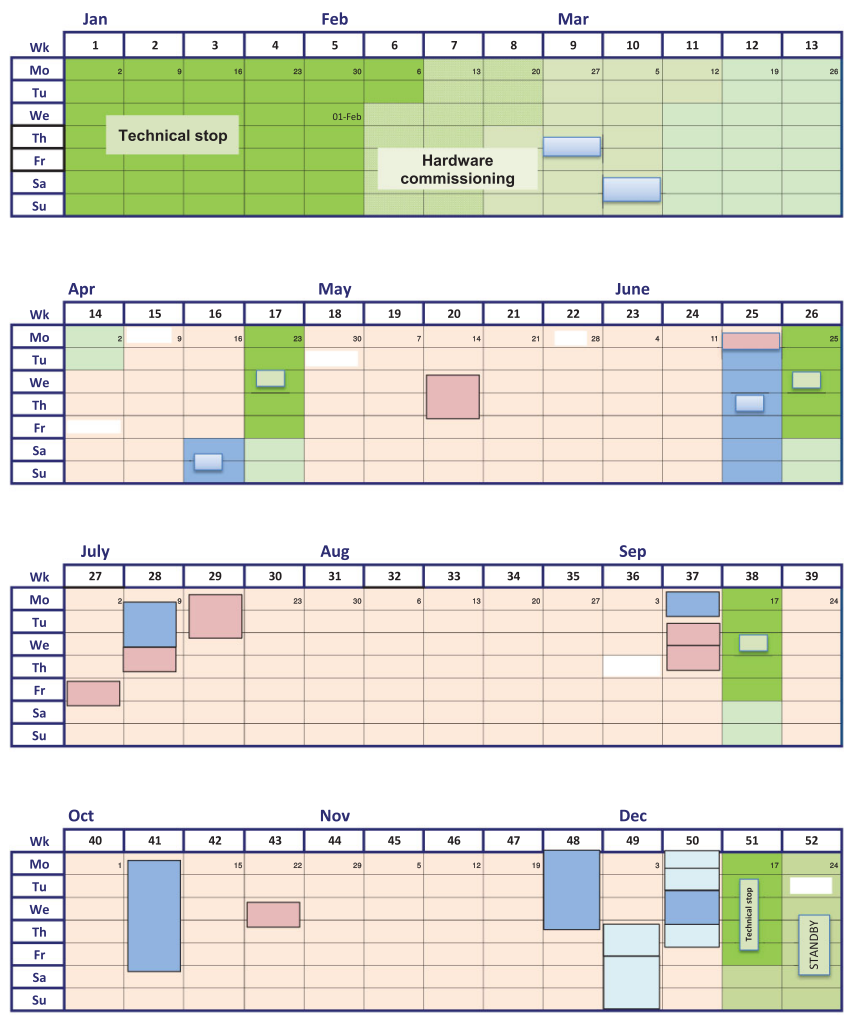

FIG. 4. LHC schedule for 2012 [65]; time scheduled for physics is marked with light orange. 


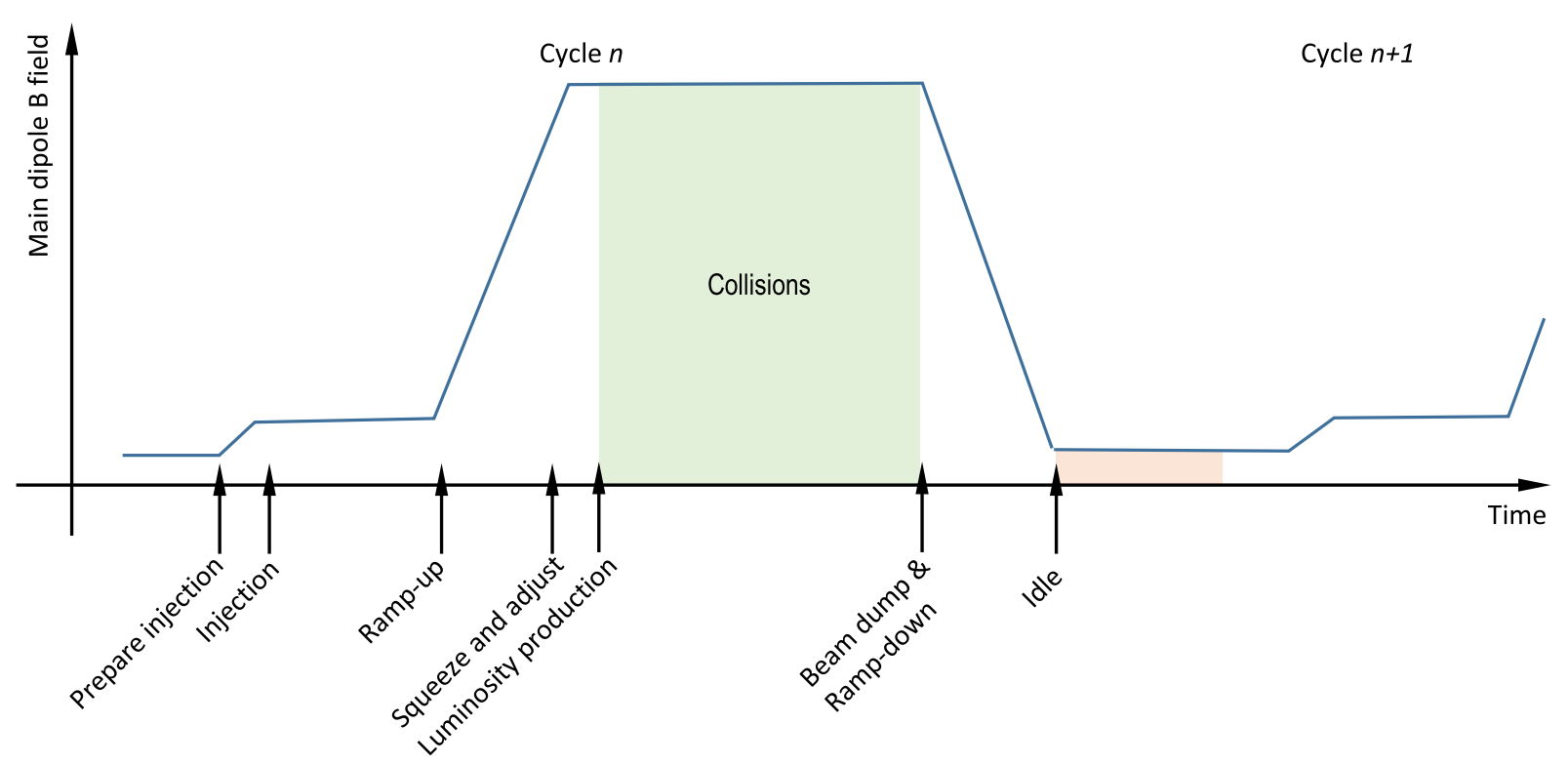

FIG. 5. Collider production cycle and cycle phases.

energy. During this phase, the radio frequency systems accelerate the particles and the magnets further ramp up their fields. (4) Squeeze and adjust: beams are prepared for collisions. (5) Luminosity production: beams are brought to collision and the experiments collect data. (6) Beam dump: beams are extracted from the rings and safely dumped. (7) Ramp-down: the magnet fields are ramped down. (8) Idle: the machine is prepared for the next cycle. The durations of injection and luminosity production depend on multiple factors. The number of bunches and machine protection constraints determine the required number of individual injections. The luminosity lifetime and average cycle duration affects the time for intentional dumps performed to optimize the luminosity production [12]. Ramp-up and ramp-down phases depend on the magnet performances, the radio-frequency system performance, the beam dynamics and last but not least, the desired beam parameters for the collision phase (energy, intensity).

Failures disrupt the standard machine cycling. Different failures cause different consequences and downtime. As an example, some failures require the magnet hysteresis standardization cycle (precycle) [66] to restore the nominal magnetic configuration before the next cycle can begin.

\section{B. LHC availability}

This work takes as a reference the 2012 LHC operation at $4 \mathrm{TeV}$, as this was so far the most stable year of luminosity production. Operation in 2010 was dedicated to commissioning. 2011 luminosity production was still limited by system optimization. 2012 is considered the first sufficiently stable reference period that is suitable as a basis for availability analysis and simulation. In 2012, 201 days were allocated for proton-proton physics [67]. During this period, the LHC was $36.5 \%$ of the time in luminosity production. Almost one third of the total time the machine was unavailable due to equipment failures. About one third of the total time was spent in cycle phases, other than luminosity production [68]. This splitting did not significantly change for the 2015 run at $6.5 \mathrm{TeV}$ (see Fig. 6), although new failure modes emerged in this year [69]. This points to a continuous learning process that a modeling and simulation setup needs to take into account.

Analysis of the downtimes in 2012 [68] and 2015 [69], as shown in Fig. 7, indicates that the cryogenic system was dominating the collider downtime. The power converters and quench protection system (QPS) [70] were in addition among the top contributors to downtime, both in 2015 and in 2012. These are among the most distributed and complex systems of the LHC, which explains the downtime contributions.

The injector chain (Linac2, PSB, PS, SPS) was also one of the key contributors to unavailability. Figure 7 shows only the injector hardware availability. Further analyses $[72,73]$ revealed that another limiting factor for availability

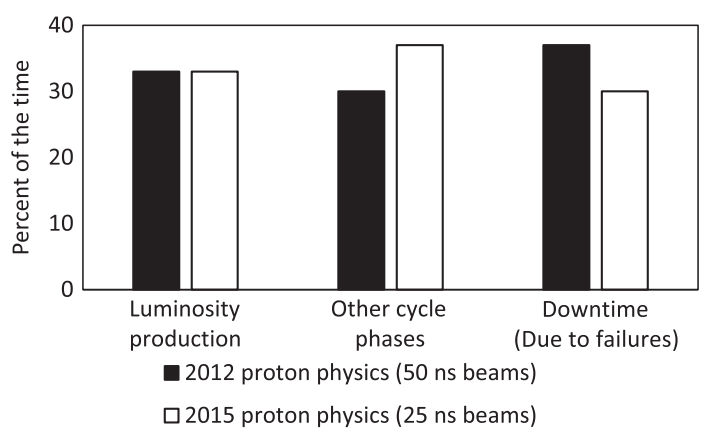

FIG. 6. LHC operational statistics for 2012 [3] and for 2015 [69]. 


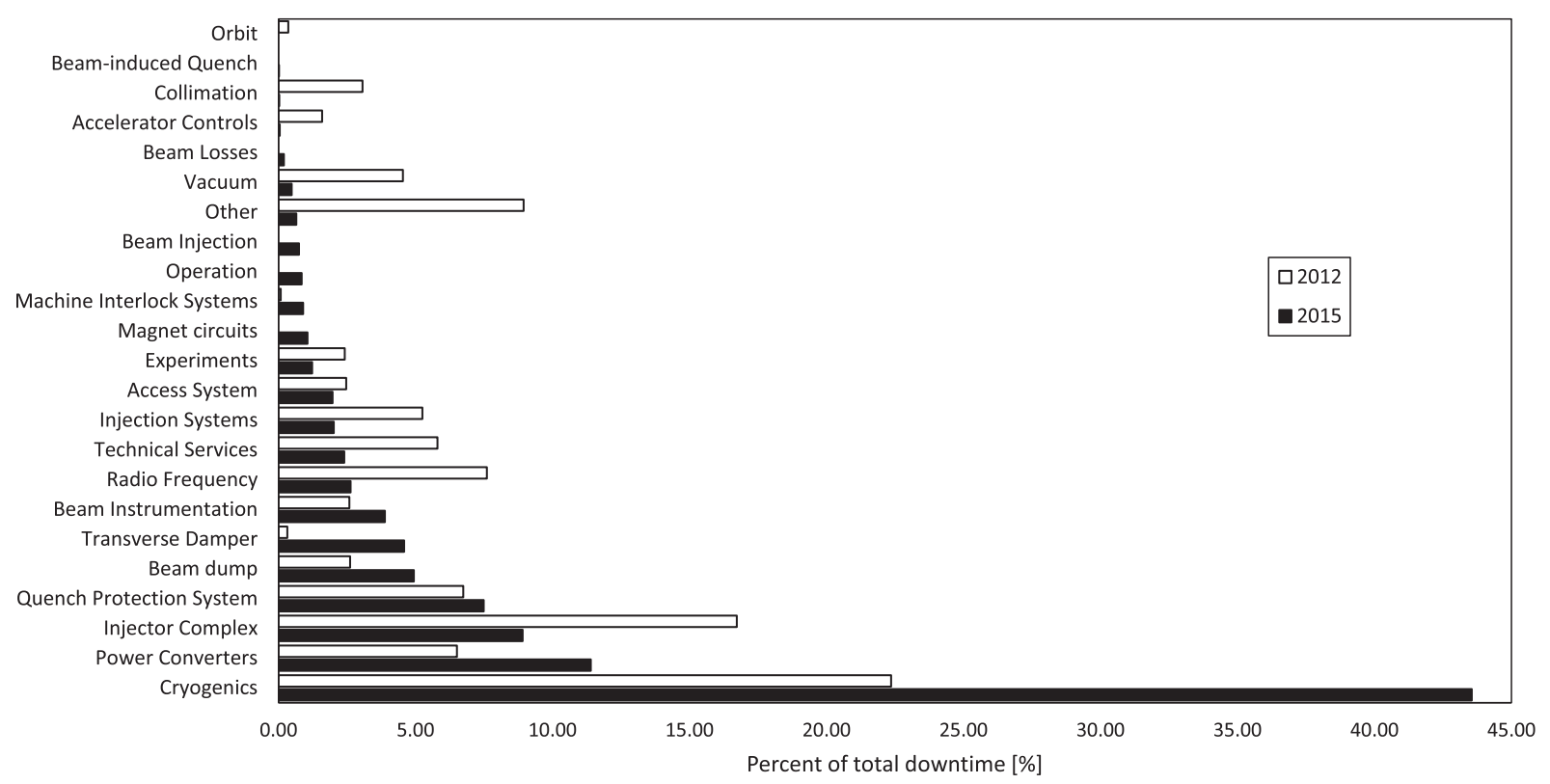

FIG. 7. LHC system downtime for 2012 and 2015 based on data from [71].

for physics is the injection phase duration. The reasons for lengthy injection phases range from beam losses due to insufficient beam quality (up to $20 \%$ rejection rate $[73,74]$ ) over injection element failures to limitations for injected intensity.

The LHC machine protection system safely discharges the high stored beam and magnet energies when requested or after a failure [75]. About $70 \%$ of the cycles reaching the luminosity production were terminated prematurely due to hardware failures or beam instabilities. The beam is dumped to prevent permanent damage of the equipment. Equipment damage can lead to significant repair times and costs.

Tight machine protection settings can result in a high number of preventive dumps. For example 79 beam related dump requests were generated at top energy in 2012 [3]. Growing experience with operating the machine allows fine-tuning of interlock settings, reducing the number of false-positive machine interlocks and thereby increasing machine availability.

\section{METHODS AND MATERIALS}

\section{A. Model requirements}

Based on the experience with LHC operation the following requirements for a suitable reliability and availability modeling and simulation environment emerge: (i) the model must include all accelerators in the chain, their subsystems and common infrastructures; (ii) the model must be incrementally extensible, since not all information about failure modes and root causes is available upfront; (iii) the model needs to include the operation modes and state dependent failure distributions; (iv) the simulation needs to implement operation schedules at all levels from multiple years down to seconds; (v) the environment must permit defining production functions for accelerator in the chain to yield meaningful and comparable availability indications; (vi) the developed environment must include a decision support model to perform sensitivity analyses of reliability and availability improvement measures for the production function. Section IV B sheds light on our approach that meets the outlined requirements.

\section{B. Modeling approach}

Figure 8 illustrates the chosen approach. Our availability model consists of interconnected machines (accelerators and transfer lines) and the necessary technical infrastructures (e.g., electricity, cryogen production, cooling supplies). Future simulations will also include maintenance and repair consumables as well as logistics and operation resources.

Each machine is composed of a number of subsystems and a fault tree describes their failure behavior. A standard structure for the top level of the fault tree ensures consistency of the modeling approach across different systems. The failure causes are divided into: (1) primary faults: failures within the defined system boundaries; (2) secondary faults: failures outside the defined system boundaries: (i) user faults: failures related to users of the system function; (ii) utility faults: failure of a utility that the given system depends upon (e.g., loss of electricity); (iii) environment faults: change in the operating environment of the system that causes a failure. The secondary faults describe dependencies between parts of the tree belonging to different systems. Deeper fault tree levels are 


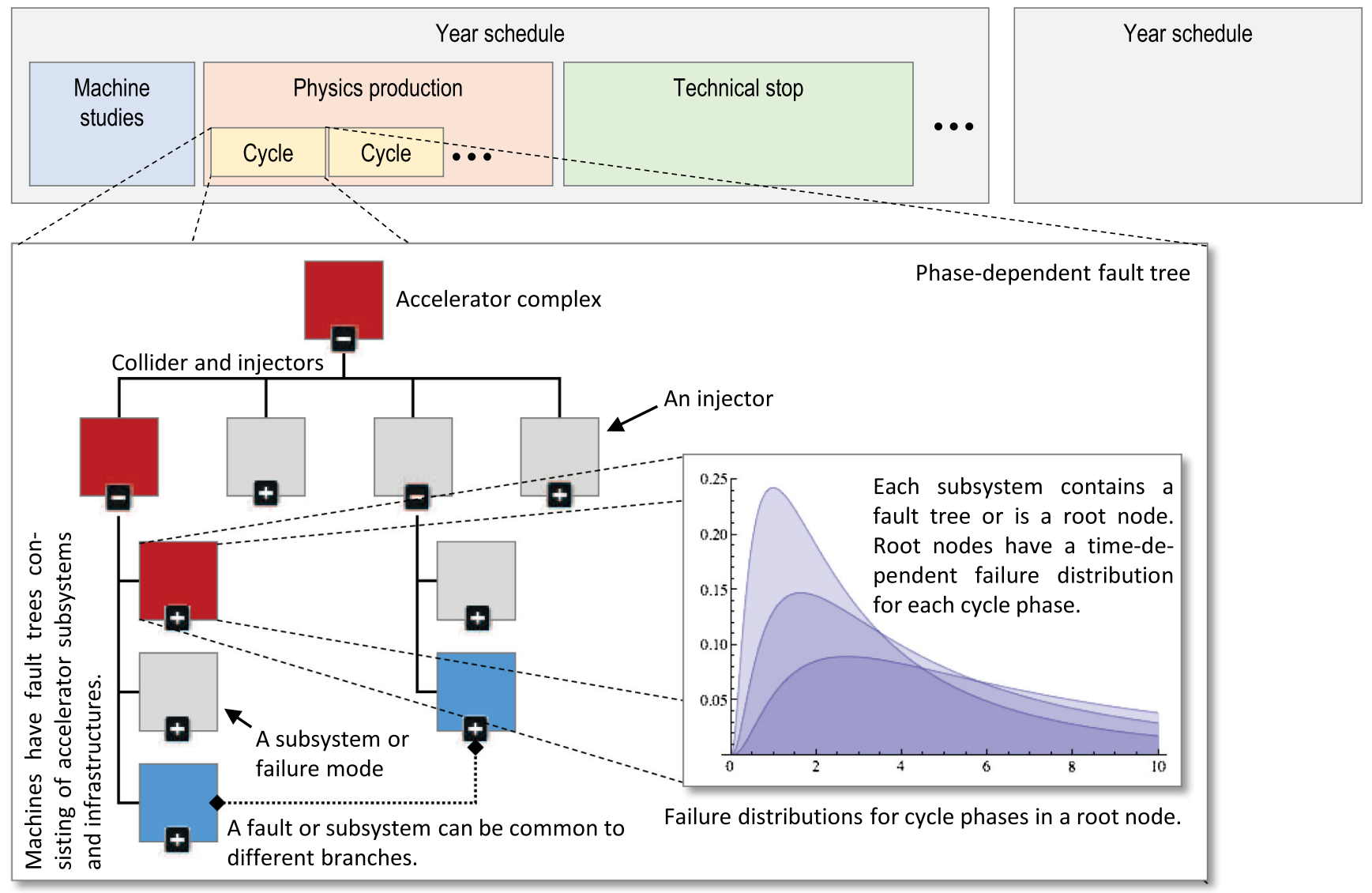

FIG. 8. The model concept with the main features.

instead reflecting the peculiarities of the different machines. Trees can be linked to each other at the node level to model common-cause failures (e.g., a single transformer station supplying electricity to the power converters of two accelerators, failure propagation from one system or one accelerator to another).

Failure events activate the fault tree nodes. In simulation, the failure event probability is calculated based on state-dependent failure distributions. The model has a set of user-defined operation states and a subsystem can have a different failure distribution associated for each operation state. The set of states is standardized for all machines in the model.

For each machine different key performance indicators (KPI) can be defined with a production function, as discussed in Sec. II A. KPIs should be used to define the machine specific availability and to compare performance of different machines.

To reproduce accelerator operations, the operation sequences form the input to the simulation. Sequences contain the state events and a holding time distribution is assigned to each state. The simulation process consists of three levels, based on Sec. III A (see Fig. 9): (1) multiyear operation schedules, including run and long shutdown (LS)
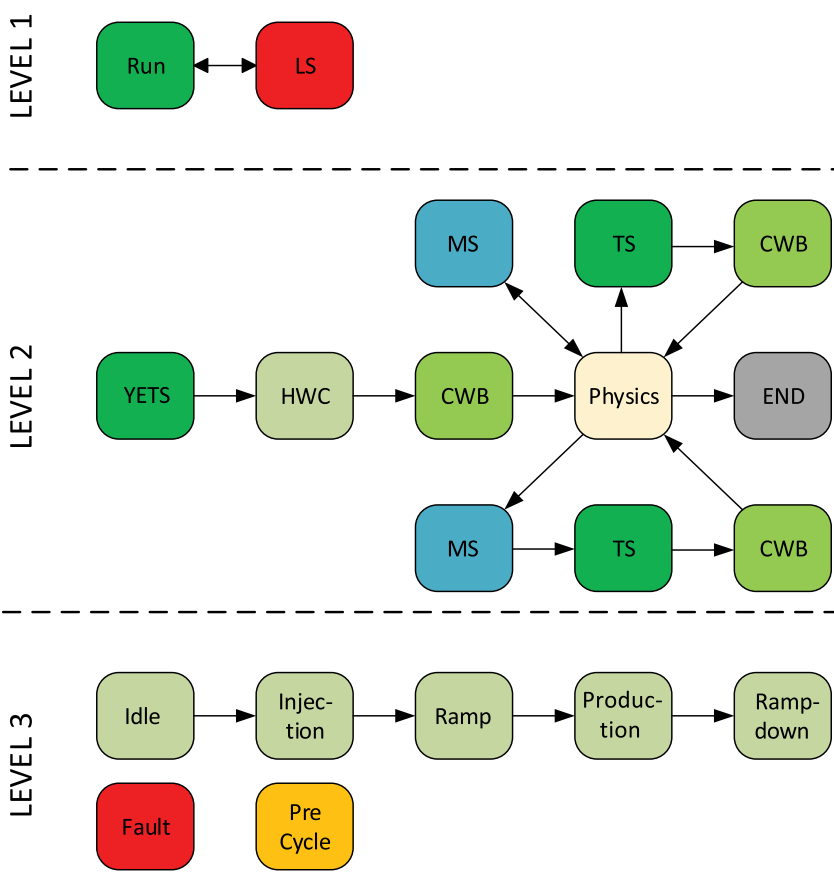

FIG. 9. Three levels of the collider operations model and normal transitions between states. 
periods; (2) yearly operation modes consisting of technical stops (TS, YETS), hardware commissioning (HWC), beam commissioning (CWB), machine studies (MS) and physics production period including intensity ramp up; (3) sequences of cycles in beam production mode, containing cycle phases (injection, ramp, luminosity production, rampdown, idle time between cycles).

The transitions between states in the sequences at each of the three levels are modeled using semi-Markov Chains [13]. This model determines the next state which is reached in case of successful or unsuccessful sequence state execution. The following logic rules describe the transitions caused by failures in different phases. Failures in the collider cause a beam dump during injection, ramp and luminosity production phases leading to a ramp-down phase. If a failure occurs in the ramp-down or in idle, the active phase does not change. The cycle completes the ramp down and waits in the idle phase, as the next injection cannot start until the failure is repaired. For certain failures a precycle is required before injection. If a failure occurs in the injector chain, while the collider is in the injection phase, it delays the operation, while during the other phases an injector failure does not affect the operation. The transition probabilities depend on the failure distributions in the fault trees.

The model is subjected to a Monte Carlo simulation approach and it requires multiple iterations to obtain meaningful results. Due to uncertainties on the input parameters, simulations aim at $10 \%$ result accuracy for luminosity production. The uncertainty relates to the relatively small data samples, which is a common problem in accelerator reliability studies (see Sec. IIC). The statistical uncertainty introduced by the Monte Carlo approach must be sufficiently small not to affect the results. This uncertainty is assessed by fitting a normal distribution to the simulation result distribution. Confidence intervals (95\%) can be derived for the mean value $\mu$.

$$
x-1.96 \frac{s}{\sqrt{N}}<\mu<x+1.96 \frac{s}{\sqrt{N}}
$$

where $x$ is the simulation mean, $s$ its standard deviation, and $N$ is number of simulation iterations. There is $95 \%$ confidence that the true value of the parameter is in the confidence interval.

\section{Simulation software tool}

The reliability and availability work unit in the FCC study assesses the fitness of a number of methods and tools for use in the particle accelerator domain in cooperation with several universities. The ELMAS [76] suite, developed by Ramentor Oy, a Finnish company that spun off Tampere University of Technology (TUT), has been adopted as the platform. The Java-based, platform-independent modeling and simulation engine implements the dynamic FTA [14] as described in this article and combines it with timedependent events. A built-in stochastic discrete event simulation engine uses this information to simulate operation schedules, considering stochasticity and delays introduced by failure, repair and maintenance events. ELMAS version 4.8, used for this study, permits specifying production functions, semi-Markov chain transition logic and conditional operation schedule specifications with userspecific Java snippets.

While the approach provides a high degree of flexibility to tailor the tool to the domain-specific needs, it breaks the concept of a single model, parametrization and simulation specification, as parts of this information is in user-supplied code. As a consequence, a collaboration between TUT, CERN, and Ramentor has been launched to develop a subsequent version of ELMAS that will permit specifying all information of the herein described model (FTA, semi-Markov chain, simulation schedules, parametrization, multisimulation of entire input parameter spaces, and sensitivity analysis specifications) without the need for programming. A separation of graphical user interface and calculation engine with an API should permit deploying the simulation engine in a cluster computing environment. Such a suite should eventually lead to an integrated, iterative approach [77] of requirements, design, RAM assessment, RAM requirements allocation, toward a cost/ availability optimized technical design for a large-scale research infrastructure.

Commercial analysis tools model typically hardware reliability and availability without integration of operation schedules, system modes and states. In house developed tools can include all features, but long-term maintainability, lack of an established user community and in-field validation of the tools are drawbacks. These shortcomings are addressed by the cooperation between CERN and Ramentor.

\section{Simulation assumptions}

We use the following assumptions to simulate the LHC luminosity production during 2012. The duration of different cycle phases is shown in Table I. Here the fixed duration is an average of the measured phase duration in 2012 data.

Luminosity production is modeled with the function:

$$
L_{\mathrm{Int}}(t)=L_{p} t_{L}\left(1-e^{-t / t_{L}}\right)
$$

TABLE I. Time lengths assumed in the simulation for each

\begin{tabular}{|c|c|c|c|c|c|}
\hline Phase & Idle & Injection & Ramp & Production & Ramp down \\
\hline Length $[\mathrm{min}]^{\mathrm{a}}$ & $180^{\mathrm{b}}$ & 52 & 49 & $576^{\mathrm{c}}$ & 35 \\
\hline
\end{tabular}
successful phase in the operation cycle. 
TABLE II. Beam parameters for simulation.

\begin{tabular}{lcc}
\hline \hline Beam $^{\mathrm{a}}$ & Peak luminosity & Luminosity lifetime \\
\hline Low intensity & $2.5 \times 10^{33} \mathrm{~cm}^{-2} \mathrm{~s}^{-1}$ & $10.5 \mathrm{~h}$ \\
Nominal intensity & $6.3 \times 10^{33} \mathrm{~cm}^{-2} \mathrm{~s}^{-1}$ & $10.0 \mathrm{~h}$ \\
\hline \hline
\end{tabular}

${ }^{\mathrm{a}}$ The parameters are averages for given periods and not related to specific filling schemes.

where $t$ is the duration of the simulated luminosity production phase, $L_{p}$ the peak luminosity and $t_{L}$ the luminosity lifetime [17]. Equation (4) assumes an exponential decay of the luminosity. We used two sets of parameters (See Table II): (1) low intensity beam to model the intensity ramp up; (2) high intensity beam for steady luminosity production. The low intensity beam is used for the first 40 days of the proton physics period.

We used the exponential distribution to generate random failure event times and repair lengths. The cumulative distribution function (CDF)

$$
\mathrm{CDF}=1-e^{-\frac{t}{E}}
$$

gives the probability that the event has occurred before time $t$. Here $E$ is the mean value of the characteristic event distribution: mean time to failure (MTTF) or mean time to repair (MTTR). Using Eq. (5) implies the assumption that failure events and repair durations are exponentially distributed. This assumption is adequate for failures times, as due to cycle phases and evolving beam parameters long term time dependencies are hard to confirm. For repair times, more detailed studies could indicate that other distributions model repair times more accurately. However, using exponential distributions does not affect the mean value of the result, the change of the distribution would only affect the result variance.

Failure statistics shown in Table III were used in the model. The available LHC hardware failure data [71] could not reliably specify the phase in which a failure occurred. These failures were thus treated as phase-independent. However, the failure and repair rates are calculated directly from the data. The occurrence rates for beam instabilities were estimated. The raw estimate was based on the fact that during 2012, beam related events caused 73 beam dumps at top energy [3]. This was the basis for the instability rate in production phase. The instabilities were assumed to be more common during injection and ramp phases leading to estimated higher occurrence rates. Also, the injector complex availability was estimated to be about $90 \%$ for LHC beams from SPS. In the statistics from [80] the injector availability for the LHC, during 2012, was more than $90 \%$. However, these statistics do not take into account if the LHC requires the beam or not. For comparison, SPS availability for fixed targets was about $80 \%$ [80]. Another source of uncertainty is that for fixed targets the statistics include the failure to provide beam with
TABLE III. Failure rates and repair times for simulation.

\begin{tabular}{lcc}
\hline \hline System $^{\mathrm{a}}$ & MTTF $[\mathrm{d}]$ & MTTR $[\mathrm{h}]$ \\
\hline Access System & 7.3 & 1.5 \\
Beam dump system & 9.8 & 1.8 \\
Beam instab. injection & $4^{\mathrm{b}}$ & 0 \\
Beam instab. ramp & $12^{\mathrm{b}}$ & 0 \\
Beam instab. production & $30^{\mathrm{b}}$ & 0 \\
Beam interlock system & 207 & 1.3 \\
Collimators & 6.8 & 1.4 \\
Controls & 9.9 & 1.2 \\
Cryogenics & 6.3 & 8.7 \\
Experiments & 6.8 & 1.4 \\
Injector Complex & $0.5^{\mathrm{b}}$ & $1.5^{\mathrm{b}}$ \\
Injection Magnets & 5.2 & 2.3 \\
Miscellaneous & 5.1 & 2.5 \\
Power Converters & 3.8 & 1.9 \\
Quench protection system & 3.7 & 1.9 \\
Radio frequency & 3.0 & 1.7 \\
Software Interlocks & 207 & 0 \\
Technical Services & 6.3 & 3.8 \\
Vacuum System & 10.7 & 3.8 \\
\hline \hline
\end{tabular}

${ }^{\mathrm{a}}$ The LHC hardware failure statistics are from CERN's failure database records for the 2012 proton physics periods [71].

${ }^{\mathrm{b}}$ Estimates.

acceptable quality, but for LHC this failure mode was excluded. So, the $90 \%$ availability value considers only the hardware availability of the injector chain. A dedicated study is currently ongoing to address this aspect [81]. The insufficient beam quality caused issues both in run 1 and run $2[73,74]$. Here this is taken into account by a longer duration of the injection phase. Sections VA and V B show the assumed failure and operation statistics, which combined with these simple estimates, give results near the observed values.

\section{RESULTS}

\section{A. Model validation}

The results of the simulation using 2012 LHC input data allow validating the approach. The distribution of the simulated integrated luminosity productions resulting from 1000 simulations is shown in Fig. 10. The mean integrated luminosity is $23.38 \mathrm{fb}^{-1}$. The measured production of $23.27 \mathrm{fb}^{-1}$ [67] is well within the set $10 \%$ accuracy goal. Equation (3) gives $\pm 0.05 \mathrm{fb}^{-1}$ confidence limits for the simulated result. That shows that 1000 simulations yield sufficient computational accuracy.

Figure 11 compares the measured and the modeled luminosity production for one model simulation. At the start of the luminosity production period, the effect of the intensity ramp up is visible through a lower production rate compared to the rest of the year.

Simplifications introduced in the model implementation explain differences between the measured and the 


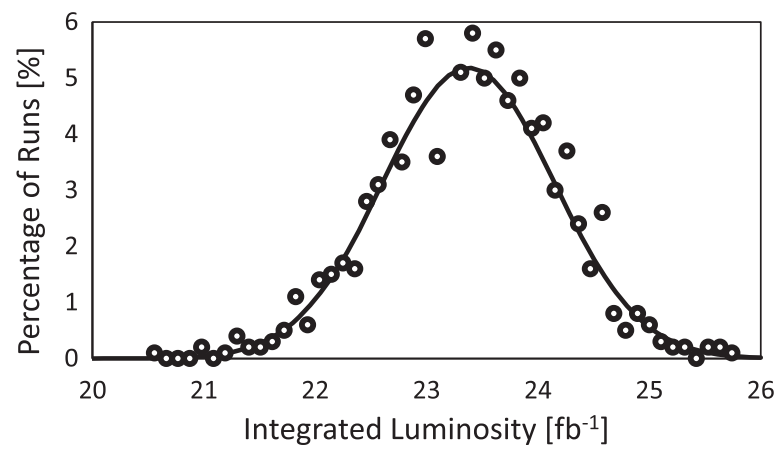

FIG. 10. Result distribution of 1000 model iterations with Gaussian fit.

simulated luminosity productions. For example, the time between technical stops is assumed to be constant in the model. Nevertheless this does not affect the availability nor the luminosity production calculations.

\section{B. Luminosity production prediction}

To assess the model prediction capabilities we simulated the 2015 luminosity production from 7.9.2015 to 3.11.2015 (58 days). During this period the LHC produced $3.9 \mathrm{fb}^{-1}$ of integrated luminosity [82].

First, to make predictions, the best estimates for operational performance published before 2015 were taken: (i) 2012 failure data from Table III; (ii) increased lengths for ramp phases for $6.5 \mathrm{TeV}$ operations (67 min for ramp up and $70 \mathrm{~min}$ for ramp down) [78]; (iii) It is assumed to have a peak luminosity of $8.1 \times 10^{33} \mathrm{~cm}^{-2} \mathrm{~s}^{-1}$ [83] and we assume an exponential decay with lifetime of $10 \mathrm{~h}$ (similar to 2012).

For a reference period of 58 days our mean result is $8.9 \mathrm{fb}^{-1}$, which is more than the actual production value of $3.9 \mathrm{fb}^{-1}$. The main reasons for this are: (1) different beam parameters used in operations compared to predictions; (2) longer injection phase (90 min) [73]. Figure 12 shows how taking these factors into account brings the result closer to the actual value. We examined four different scenarios: (1) with the 2014 input values from [83]; (2) with theoretical beam parameters for the period: peak luminosity
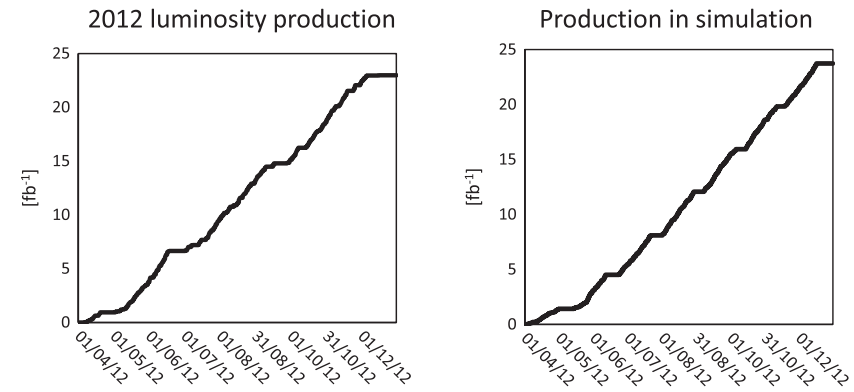

FIG. 11. Actual LHC integrated luminosity production during 2012 compared to production in one model simulation.

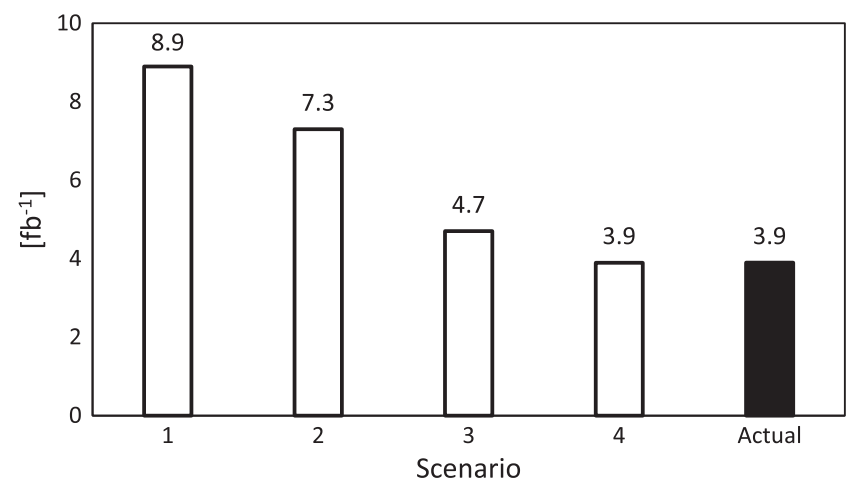

FIG. 12. Predictions for integrated luminosity production in the LHC during 2015 reference period compared to actual value.

of $4.5 \times 10^{33} \mathrm{~cm}^{-2} \mathrm{~s}^{-1}$ and luminosity lifetime of $30-60 \mathrm{~h}$ [84]; (3) with actual average peak luminosity value for the period $\left(2.9 \times 10^{33} \mathrm{~cm}^{-2} \mathrm{~s}^{-1}\right)$ [82]; (4) with the actual average peak luminosity value and $90 \mathrm{~min}$. long injection phase.

The year 2015 followed two years of shutdown. This caused uncertainties and challenges for operations and made performance predictions difficult. For example, the peak luminosity value was overestimated, due to: (i) delayed start of the proton-physics and a special physics run during the studied period that limited the luminosity production [85]; (ii) technical difficulties encountered in August related to radiation effects [86]; (iii) performance after the long shutdown was limited by deconditioning effects (e.g. electron cloud [87] and interaction of the beam with dust particles (unidentified falling objects (UFO)) [88]). The main reasons for the longer injections were injection intensity limitations due to electron cloud and insufficient beam quality [73].

\section{Multiyear scenario example}

Our approach is capable of modeling different schedules. Production stops can greatly affect the overall amount of integrated luminosity. This can be demonstrated with a simple example using the LHC operational data [82]. In this hypothetical scenario we assume reduction of the nonproductive time. The year 2010 was dominated by commissioning. However, luminosity was produced during 2015, although it was the first year after the first long shutdown (LS1) [89]. Here we assume the same to be true for 2010. In addition it is assumed that LS1 would have stopped already in 2014. Stopping the LS1 in 2014 is an unrealistic assumption, as the LS1 was triggered by the need to consolidate the magnet interconnections to allow the LHC to operate at the design energy of $14 \mathrm{TeV}$ [90].

Thanks to the changes the years 2011 and 2015 can be used for luminosity production. In this scenario the LHC could have produced double the amount of current integrated luminosity [91] as shown by Fig. 13. The scenario is formed just by rearranging the operation data. Creating 


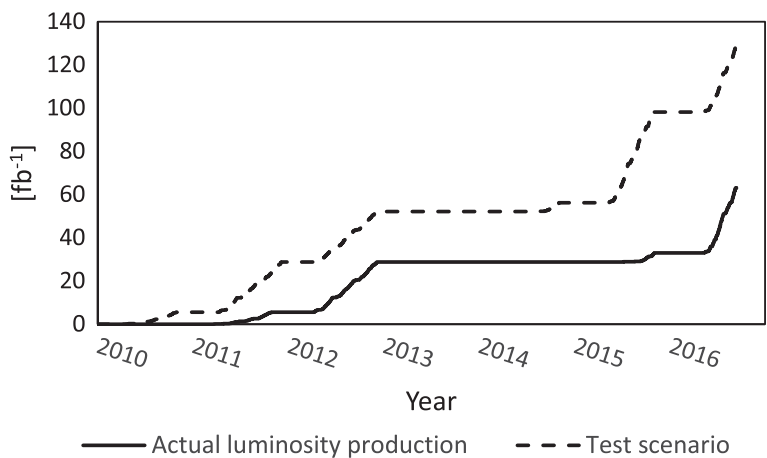

FIG. 13. The actual LHC production compared to a scenario with less nonproductive time.

more complex scenarios with changing schedules, production functions, failure rates or recovery times requires a simulation model.

\section{Sensitivity analysis}

We created an example scenario to show how our model can be used to assess the sensitivity of luminosity production on the input parameters. As operating conditions change between years and runs, it is important to be able to extrapolate the behavior of failure causes and assess their impact on operations.

In 2015 interaction of the beam with dust particles (unidentified falling objects (UFO) [88]) caused 21 beam dumps of which 3 caused a quench in the superconducting magnets [69]. This failure mode was first observed in 2015 when the beam energy was increased to $6.5 \mathrm{TeV}$. The UFO occurrence rate and consequences correlate with the beam energy. Each UFO has a probability to produce beam losses leading to quench.

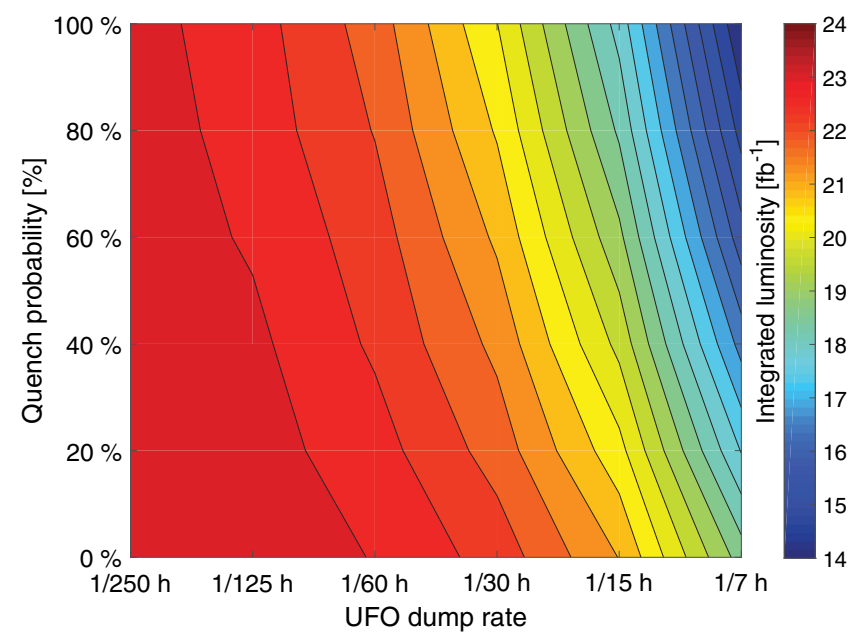

FIG. 14. Sensitivity analysis of luminosity production to UFO dump rate and quench probability, based on LHC 2012 operational data. Quench probability is the probability of a quench to occur in each UFO dump.
We performed a what-if scenario on the 2012 data, introducing quenches caused by UFOs. The amount of luminosity production is calculated with different UFO dump rates and quench probabilities. We assumed that a quench causes nine hours of downtime.

The results in Fig. 14 show that the integrated luminosity production depends strongly on the UFO dump rate whereas it is less sensitive to the quench probability. Each dump causes the cycle to be reexecuted. The downtime increases with higher quench probability. This is due to the fact that cryogenic conditions need to be recovered after a quench.

This sensitivity analysis confirms the decision taken in 2015 and 2016, when the beam loss monitor thresholds were increased to limit the UFO dump rate while accepting higher beam losses [88]. Our results indicate that decreasing the dump rates can increase the luminosity production, even if the downtime associated to a dump would be longer.

\section{DISCUSSION}

The work carried out so far indicates that planning for reliability and availability is severely limited by the availability of high-quality operation monitoring information. Implementation of an effective model is mostly hampered by the lack of data integration among different machines and subsystems, limited granularity for failure events' root causes and related operation modes.

Consequently, the work on reliability and availability modeling and simulation in the scope of the future circular collider study has been reinforced: a comprehensive training program for about 100 engineers working in different domains has been launched to promote a coherent language and set of methods and tools [92]. The aim is to gradually build a consistent set of fault trees and a data source of subsystem reliability information for the continued work. A data analytics infrastructure, integrating numerous heterogeneous accelerator monitoring databases, is being established to have a single source of high-quality fault and operation monitoring information. The infrastructure is based on modern column-oriented database models and it can provide the input parameters to the simulation models [81].

However, there will always be uncertainty on the input values. Here we took into account uncertainty caused by the Monte Carlo approach. Taking into account the uncertainty of the input values requires determining confidence bounds for individual inputs. This creates a multidimensional space of possible input combinations. Various techniques exist to determine the uncertainty in the results, when input confidence bounds are defined, e.g., Latin hypercube sampling is a popular option for this [93]. In our case, a practical application of this will require deploying the simulation engine in a cluster computing setup with input parameter database.

The planned application of our model is comparing various scenarios, like shown in Sec. V. These scenarios can have different failure rates, recovery times, production 
functions, operational schedules, and operation and capital expenditures. Creating these scenarios, when new technology is involved, depends on expert estimates. Thus, estimating the exact uncertainty in the inputs is difficult. However, what is important is the relative comparison of different scenarios. For this purpose, understanding the exact uncertainty is less important.

Our availability study focuses on frequently occurring failures. Individually these failures have relatively limited consequences, but their occurrence rates hinder the operations. Our approach, as is, cannot be applied to rare failures with severe consequences. For example, such incident occurred in LHC in 2008 due to defective joint between superconducting cables delaying the physics program for more than a year [94]. Prevention of severe failures requires detailed studies of specific systems. This is already well-established activity in the accelerator community. For example, the need for reliable machine protection systems was identified during LHC design phase [75] and a great care is put into prevention of failures that can affect personnel safety [95].

\section{CONCLUSIONS AND FUTURE WORK}

This article introduces a novel approach to model and efficiently forecast the reliability and availability of entire particle accelerator infrastructures based on industrial practices and tools. A proof-of-concept application to the Large Hadron Collider infrastructure revealed the value of applying a process-oriented approach to model a complex of particle accelerators, its infrastructures and operation environment. A combination of stochastic fault-trees with domain-specific production functions and multilevel semiMarkov chains is well suited to perform sensitivity analyses of alternate designs, reliability improvements of existing systems, and different operation and maintenance schedules. Most notably, this work revealed the importance to base any reliability and availability planning for upgrades and future green-field projects on the data gathered from multiple years of operating machines. This data does not yet exist from the LHC in such quantities. Thus, deriving credible availability and reliability goals for FCC will require LHC operations data from several more years.

This work presented the concept and case study of the LHC to validate the model and the accuracy of the simulation. The next step is to include further subsystems in the model, notably all machines along the injector chain and key infrastructures such as electricity, cryogenics and vacuum. Concerning machine schedules, the study will explore the impact of varying yearly and multiyear operation schedules as well as the impact of logistics (transport and spare part policies) on the overall luminosity production.

This initiative leverages synergies between the LHC operations and the HL-LHC upgrade projects: both activities can potentially profit directly from a work that aims at modeling a future, $100 \mathrm{~km}$ long particle collider with its injector infrastructure. The established modeling and simulation infrastructure can also be used to test the cost/value relation of reliability and availability enhancing scenarios for the operating collider infrastructure and can help optimize the designs for the forthcoming machine upgrades.

\section{ACKNOWLEDGMENTS}

Ramentor Oy's ELMAS software was used as the platform for the model. The authors thank the company for the chance to use the software and for support during the model development. Special thanks to Eric Coatanéa, Tobias Griesemer, Volker Mertens, Odei Rey Orozco, and Rüdiger Schmidt for providing references and corrections to the text. This project and its achievements are part of the global future circular collider study hosted by CERN. The FCC study has received funding from the European Union's Horizon 2020 research and innovation programme under Grant Agreement No. 654305 (EuroCirCol). In Finland, the work for the FCC availability study has received funding from Ramentor $\mathrm{Oy}$ and Tampere University of Technology.

[1] M. Benedikt and F. Zimmermann, The Future Circular Collider study, CERN Courier 54, 16 (2014).

[2] P. Collier, Availability, Operation \& Energy, in Future Circular Collider Study Kickoff Meeting (University of Geneva, Geneva, 2014), https://indico.cern.ch/event/282344/ contributions/1630773/.

[3] A. Apollonio, Ph. D. thesis, Vienna University of Technology, 2015.

[4] A. Apollonio, M. Jonker, R. Schmidt, B. Todd, S. Wagner, D. Wollmann, and M. Zerlauth, HL-LHC: Integrated Luminosity and Availability, in Proceedings of the 4th International Particle Accelerator Conference, IPAC2013, Shanghai, China, 2013 (JACoW, Shanghai, 2013), p. 1352 .

[5] B. Todd, A. Apollonio, L. Ponce, and C. Roderick, Accelerator Fault Tracker \& Availability Working Group - Where Do We Go From Here?, in LHC Performance Workshop (Chamonix, 2016), https://indico.cern.ch/event/448109/ contributions/1942065/.

[6] W. Vesely, F. Goldberg, N. Roberts, and D. Haasl, Fault Tree Handbook (U.S. Nuclear Regulatory Commission, Washington, 1981).

[7] R. Rubinstein and B. Melamed, Modern Simulation and Modeling (Wiley, New York, 1998).

[8] M. Rausand and A. Høyland, System Reliability Theory: Models, Statistical Methods and Applications (Wiley, New York, 2004).

[9] B. Bertsche, Reliability in Automotive and Mechanical Engineering (Springer, New York, 2008), doi: 10.1007/ 978-3-540-34282-3.

[10] J. Toes, ARIES approved by European Commission, Accelerating News is a quarterly online publication for the accelerator community, Accelerating News (2016), issue 18. 
[11] F. Marcastel, CERN Technical Report No. OPEN-PHOCHART-2013-001, 2013.

[12] W. Herr and B. Muratori, Concept of luminosity, in CAS-CERN Accelerator School: Intermediate Course on Accelerator Physics, Cern accelerator school at Zeuthen, Germany, 2003 (CERN, Geneva, 2006), pp. 361-377, http://cds.cern.ch/record/941318.

[13] B. Bertsche, Maintenance and Reliability, in [9], Chap. 10, p. 338, doi: 10.1007/978-3-540-34282-3_10.

[14] J.-P. Penttinen and T. Lehtinen, Advanced Fault Tree Analysis for Improved Quality and Risk Assessment, in Proceedings of the 10th World Congress on Engineering Asset Management (WCEAM 2015), Lecture Notes in Mechanical Engineering (Springer Publishing, New York, 2016),p.471, https://doi.org/10.1007/978-3-319-27064-7_45.

[15] International Electrotechnical Commission, International electrotechnical vocabulary-Part 192: Dependability, IEC 60050-192 (IEC, 2015) international standard.

[16] T. Kanti Agustiady and E.-A. Cudney, Equipment Effectiveness, Total Productive Maintenance: Strategies and Implementation Guide (CRC Press, Boca Raton, 2015), Chap. 6, p. 111.

[17] J. Wenninger, CERN Technical Report No. CERN-ATSNote-2013-033 PERF, 2013, internal CERN document, remarks on exponential decay of luminosity assumption available in [2], Chap. 7.1.1 p. 371 and in [3], Chap. 6.1.4 pp. 93-97.

[18] M. Benedikt, D. Schulte, and F. Zimmermann, Optimizing integrated luminosity of future hadron colliders, Phys. Rev. ST Accel. Beams 18, 101002 (2015).

[19] B. Bertsche, FMEA - Failure Mode and Effects Analysis, in [9], Chap. 4, p. 98, doi: 10.1007/978-3-540-34282-3_4.

[20] W. Vesely, F. Goldberg, N. Roberts, and D. Haasl, Fault Tree Analysis-Basic Concepts, in [6], Chap. 3.

[21] M. Rausand and A. Høyland, Reliability Block Diagrams, in [8], Chap. 3.10, p. 118.

[22] B. Bertsche, Lifetime Distributions for Reliability Description, in [9], Chap. 2.2, p. 35, doi: 10.1007/978-3-54034282-3_2.

[23] L. Hardy, Accelerator Reliability - Availability, in Proceedings of the 8th European Particle Accelerator Conference, Paris, 2002 (EPS-IGA and CERN, Geneva, 2002), p. 149.

[24] O. Brüning, S. Oliver, P. Collier, P. Lebrun, S. Myers, R. Ostojic, J. Poole, and P. Proudlock, Performance goals, LHC Design Report (CERN, Geneva, 2004) Chap. 3.1.1, p. 21, doi: 10.5170/CERN-2004-003-V-1.

[25] K. T. Dixon and J. Franciscovich, SSC Accelerator Availability Allocation, in Supercollider 3 (Springer, New York, 1991), p. 1109, doi: 10.1007/978-1-4615-3746-5_102.

[26] Y. Cho, Utilisation and Reliability of High Power Proton Accelerators, in Workshop Proceedings, Aix-en-Provence, France, 22-24 November 1999 (OECD Nuclear Energy Agency, Aix-en-Provence, 1999), p. 71, doi: 10.1787/ 9789264194847-en.

[27] T. Himel, J. Nelson, N. Phinney, and M. Ross, Availability and Reliability Issues for ILC, in Proceedings of the 22nd Particle Accelerator Conference, PAC-2007, Albuquerque, NM (IEEE, New York, 2007), p. 1966, doi: 10.1109/ PAC.2007.4441325.
[28] J. Biarrotte, A. Mueller, H. Klein, P. Pierini, and D. Vandeplassche, Accelerator reference design for the MYRRHA European ADS demonstrator, in Proceedings of the 25th International Linear Accelerator Conference, LINAC-2010, Tsukuba, Japan (KEK, Tsukuba, 2010), p. 440.

[29] M. Aicheler, P. Burrows, M. Draper, T. Garvey, P. Lebrun, K. Peach, N. Phinney, H. Schmickler, D. Schulte, and N. Toge, Power and energy consumption for staged construction and operation scenario, A Multi-TeV linear collider based on CLIC technology: CLIC Conceptual Design Report (CERN, Geneva, 2012), Chap. 9.4.5, p. 789, doi: 10.5170/CERN-2012-007.

[30] E. Bargalló Font, Ph.D. thesis, Polytechnic University of Catalonia, 2014

[31] A. Apollonio, S. Gabourin, C. Martin, B. Mikulec, B. Puccio, J. L. Sanches Alvarez, D. Wollmann, and M. Zerlauth, Availability Studies for LINAC4 and Machine Protection Requirements for LINAC4 Commissioning, in IPAC2014: Proceedings of the 5th International Particle Accelerator Conference (JACoW, Dresden, 2014), p. 3807, http://accelconf.web.cern.ch/AccelConf/IPAC2014/index .htm.

[32] G. Apollinari, O. Brüning, T. Nakamoto, and L. Rossi, High Luminosity Large Hadron Collider HL-LHC, in $H L$ LHC Preliminary Design Report, CERN-ACC-2014-0300 (CERN, 2014), Chap. 1, pp. 33-34.

[33] The CEPC-SPPC Study Group, Machine Layout and Performance, in CEPC-SppC Preliminary Conceptual Design Report, Vol. II: Accelerator (Institute of High Energy Physics, CAS, Beijing, China, 2015), Chap. 3, p. 39.

[34] E. Bargalló, R. Andersson, A. Nordt, A. De Isusi, E. Pitcher, and K. H. Andersen, ESS Reliability and Availability Approach, in IPAC 2015: Proceedings of the 6th International Particle Accelerator Conference (JACoW, Richmond, 2015), p. 1033, https://jacowfs.jlab.org/conf/ proceedings/IPAC2015/.

[35] R.W. Hamm, Industrial Accelerators, Rev. Accel. Sci. Techol. 01, 163 (2008).

[36] A. Lüdeke, Operation event logging system of the Swiss Light Source, Phys. Rev. ST Accel. Beams 12, 024701 (2009).

[37] X. Deletoille, H. Rozelot, P. Betinelli, C. Herbeaux, B. Cortes, T. Didier, and L. Manciet, Machine operation and daily maintenance management in SOLEIL, in 2nd Asset and Maintenance Management Workshop - AMMW 2013 (CERN, Geneva, 2013), http://indico.cern.ch/event/233494/ contributions/497822/.

[38] A. Peeters, J.P. Grutters, M. Pijls-Johannesma, S. Reimoser, D. De Ruysscher, J. L. Severens, M. A. Joore, and P. Lambin, How costly is particle therapy? Cost analysis of external beam radiotherapy with carbon-ions, protons and photons, Radiotherapy and oncology 95, 45 (2010).

[39] O. Rey Orozco, E. Bargalló, A. Nordt, A. Apollonio, and R. Schmidt, Reliability and Availability Modeling for Accelerator Driven Facilities, in IPAC2014: Proceedings of the 5th International Particle Accelerator Conference (JACoW, Dresden, 2014), pp. 3803-3806, http:// accelconf.web.cern.ch/AccelConf/IPAC2014/index.htm. 
[40] E. S. McCrory and P. W. Lucas, A Model of the Fermilab Collider for Optimization of Performance, in Proceedings of the Particle Accelerator Conference, Dallas, 1995 (IEEE, New York, 1995), p. 449.

[41] E.S. McCrory, Monte Carlo of Tevatron Operations, Including the Recycler, in Proceedings of the 21st Particle Accelerator Conference, Knoxville, TN, 2005 (IEEE, Piscataway, 2005), p. 2479.

[42] I. Balan, Ph.D. thesis, Karlsruhe Institute of Technology, 2005.

[43] C. Tapia, J. Dies, J. Abal, A. Ibarra, and J. M. Arroyo, Exploration of reliability databases and comparison of former IFMIF's results, Fusion Eng. Des. 86, 2726 (2011).

[44] P. J. Sureda Pastor, Master's thesis, Polytechnic University of Catalonia, 2013.

[45] N. Phinney, T. Himel, P. Czarapata, H. Edwards, and M. Huening, Reliability simulations for a linear collider, in Proceedings of the 9th European Particle Accelerator Conference, Lucerne, 2004 (EPS-AG, Lucerne, 2004), p. 857, http://accelconf.web.cern.ch/AccelConf/e04/.

[46] See http://www.accelerator-reliability.org/software/.

[47] B. Carluec and B. Giraud, The European Project PDS-XADS - "Preliminary Design Studies of an eXperimental Accelerator-Driven System", Euradwaste'04 - Radioactive Waste Management - Community Policy and Research Initiatives (European Commission Community Research and Development Information Service, Luxembourg, 2004), http://cordis.europa.eu/ fp6-euratom/ev_euradwaste04_proceedings.htm.

[48] L. Burgazzi and P. Pierini, Reliability studies of a highpower proton accelerator for accelerator-driven system applications for nuclear waste transmutation, Reliability Engineering and System Safety 92, 449 (2007).

[49] PTC, "What's New in PTC Windchill@ Quality Solutions 10.2? (2013).

[50] J. Sörman and G. von Feilitzen, RiskSpectrum Magazine, Special RiskSpectrum PSA Edition (Relcon Scandpower AB, Sundbyberg, 2008).

[51] A. Pitigoi and P. Fernández Ramos, Modeling High-Power Accelerators Reliability - Reliability of SNS LINAC (SNSORNL); Reliability Modelling for MAX LINAC (Myrrha Project), in 11th International Topical Meeting on Nuclear Applications of Accelerators (AccApp 2013) (SCK-CEN, Bruges, 2013).

[52] O. Rey Orozco, A. Apollonio, M. Jonker, and M. Paoluzzi, Dependability studies for CERN PS Booster RF system upgrade, in IPAC2016: Proceedings of the 7th International Particle Accelerator Conference (JACoW, Busan, 2016), p. 4159, http://accelconf.web.cern.ch/AccelConf/ipac2016/.

[53] Isograph Ltd, Isograph, http://www.isograph.com, accessed: 2016-07-01.

[54] United States Department of Defence, Military Handbook Reliability Prediction of Electronic Equipment (1991).

[55] C. Piaszczyk, Operational Experiences at Existing Accelerator Facilities, in Utilisation and Reliability of High Power Proton Accelerators Workshop Proceedings, Mito, Japan, 13-15 October 1998 (OECD Nuclear Energy Agency, Mito, Japan, 1998), p. 163, doi: 10.1787/ 9789264181168-en.
[56] C. Piaszczyk, Accelerator reliability database, in Proceedings of the 18th Particle Accelerator Conference, New York, 1999 (IEEE, New York, 1999), p. 1465, doi: 10.1109/ pac.1999.795583.

[57] J. Galambos, SNS performance and the next generation of high power accelerators, in Proceedings of the 25th Particle Accelerator Conference, PAC-2013, Pasadena, 2013 (IEEE, New York, 2013), p. 1443.

[58] A. Lüdeke, M. Bieler, R. H. A. Farias, S. Krecic, R. Müller, M. Pont, and M. Takao, Common operation metrics for storage ring light sources, Phys. Rev. ST Accel. Beams 19, 082802 (2016).

[59] J. Moubray, Reliability Centered Maintenance, 2nd ed. (Industrial Press Inc., New York, 2005).

[60] X. Jia and A. Christer, Case Experience Comparing the RCM Approach to Plant Maintenance with Modelling Approach, in Case Studies in Reliability and Maintenance (Wiley, New York, 2003), Chap. 21, p. 477.

[61] M. Bernardini, Framework schedule from YETS 2015 to LS3, in Joint LIU /HL-LHC meeting (CERN, Geneva, 2015).

[62] E. Bravin, LHC Report: LHC hit the target!, CERN Bulletin (2016), issue No. 36-37/ 2016.

[63] J. Wenninger, LHC operation in 2015 and prospects for the future, in Proceedings of the 51st RENCONTRES DE MORIOND, 2016 Electroweak Interactions and Unified Theories (ARISF, La Thuile, 2016), pp. 239-245.

[64] J. Wenninger, Machine Protection and Operation for LHC, CERN Yellow Rep. 2, 377 (2016).

[65] CERN Beams Department, 2012 LHC schedule v 2.6 (CERN, Geneva, Switzerland, 2012).

[66] L. Bottura, M. Lamont, E. Todesco, W. Venturini Delsolaro, and R. Wolf, CERN Technical Report No. CERN-ATS-2010-174, 2010.

[67] A. Macpherson, CERN Report No. CERN-ATS-2013-045, 2012, p. 1.

[68] B. Todd, A. Apollonio, A. Macpherson, J. Uythoven, and D. Wollmann, CERN Report No. CERN-ATS-2013-045, 2012, p. 7.

[69] A. Apollonio, B. Auchmann, L. Ponce, C. Roderick, R. Schmidt, M. Solfaroli Camillocci, B. Todd, D. Wollmann, and M. Zerlauth, CERN Report No. CERN-ACC-2015376, 2015, p. 19.

[70] L. Coull, D. Hagedorn, V. Remondino, and F. RodriguezMateos, LHC magnet quench protection system, IEEE Trans. Magn. 30 (1994).

[71] CERN, Accelerator fault tracking, aft.cern.ch (Accessed 2015), an internal website publishing LHC failure data.

[72] M. Solfaroli Camillocci, CERN Report No. CERN-ACC2015-376, 2015, p. 45.

[73] D. Jacquet, Injection, CERN Report No. CERN-ACC2015-376, 2015, p. 49.

[74] L. Norderhaug Drøsdal, Ph.D. thesis, University of Oslo, 2015.

[75] R. Schmidt, R. Assmann, E. Carlier, B. Dehning, R. Denz, B. Goddard, E. B. Holzer, V. Kain, B. Puccio, B. Todd, J. Uythoven, J. Wenninger, and M. Zerlauth, Protection of the CERN Large Hadron Collider, New J. Phys. 8, 290 (2006).

[76] ELMAS = Event, Logic, Modeling, Analysis, Software, see http://www.ramentor.com/products/elmas. 
[77] P.-E. Hagmark and S. Virtanen, Simulation and calculation of reliability performance and maintenance costs, in IEEE 2007 Annual Reliability and Maintainability Symposium (IEEE, New York, 2007), p. 34, doi: 10.1109/ RAMS.2007.328102.

[78] M. Solfaroli Camillocci, CERN Report No. CERN-ATS2013-045, 2012, p. 23.

[79] M. Lamont, CERN Report No. CERN-2014-006, 2013, p. 139, doi: 10.5170/CERN-2014-006.139.

[80] K. Cornelis, SPS availability, in The Workshop on Machine Availability and Dependability for Post-LS1 LHC (CERN, Geneva, 2013).

[81] V. Begy, A. Apollonio, J. Gutleber, M. Martin Marquez, A. Niemi, J.-P. Penttinen, E. Rogova, A. Romero Marin, and P. Sollander, Big Data Analytics for Future Circular Collider Reliability and Availability Studies, in The 22nd International Conference on Computing in High Energy and Nuclear Physics, CHEP 2016, was hosted by SLAC and LBNL (San Francisco, 2016), https://indico.cern .ch/event/505613/contributions/2228319/.

[82] CERN, Accelerator performance and statistics, lhcstatistics.web.cern.ch (Accessed 2016), an internal website publishing LHC operational data.

[83] J. Wenninger, Strategy for Run 2, in Proceedings of the Chamonix 2014 Workshop on LHC Performance, CERN Yellow Reports, Vol. 2 (Chamonix, 2015), pp. 129-133, doi: 10.5170/CERN-2015-002.129.

[84] F. Antoniou, G. Arduini, M. Hostettler, G. Iadarola, Y. Papaphilippou, S. Papadopoulou, G. Papotti, and G. Trad, CERN Report No. CERN-ACC-2015-376, p. 77.

[85] G. Papotti, Lessons from LHC operation in 2015, in LHC Performance Workshop (Chamonix, 2016), https://indico .cern.ch/event/448109/contributions/1942059/.
[86] M. Pojer, CERN Report No. CERN-ACC-2015-376, 2015, p. 29.

[87] G. Iadarola, H. Bartosik, K. Li, L. Mether, A. Romano, G. Rumolo, and M. Schenk, CERN Report No. CERN-ACC2015-376, 2015, p. 101.

[88] B. Auchmann, J. Ghini, L. Grob, G. Iadarola, A. Lechner, and G. Papotti, CERN Report No. CERN-ACC-2015-376, 2015, p. 81.

[89] First long shutdown for the LHC was from February 2013 to December 2014.

[90] F. Bordry, S. Baird, K. Foraz, A. Perrot, R. Saban, and J. Tock, The first long shutdown (LS1) for the LHC, in Proceedings of the 4th International Particle Accelerator Conference, IPAC-2013, Shanghai, China, 2013 (JACoW, Shanghai, 2013), p. 44.

[91] Integrated luminosity in scenario is $128 \mathrm{fb}^{-1}$. Current produced integrated luminosity is $63 \mathrm{fb}^{-1} 15.9 .2016$, based on [82].

[92] J. Gutleber, Accelerator Reliability and Availability Training, Accelerating News (2016), issue 16.

[93] J. Helton, J. Johnson, C. Sallaberry, and C. Storlie, Survey of sampling-based methods for uncertainty and sensitivity analysis, Reliability Engineering System Safety 91, 1175 (2006).

[94] L. Rossi, Superconductivity: its role, its success and its setbacks in the Large Hadron Collider of CERN, Supercond. Sci. Technol. 23, 034001 (2010).

[95] P. Ninin, IEC 61508 experience for the development of the LHC functional safety systems and future perspective, in ICALEPCS 2009 Contributions to the Proceedings (JACoW, Kobe, 2009), p. 400, http://accelconf.web.cern .ch/Accelconf/icalepcs2009/index.htm. 